for: Amgen, Galapagos, UCB, Consultant of: Eli Lilly, Johnson \&Johnson, Novartis Galapagos, UCB, Grant/research support from: Celgene, Elisa Gremese: None declared, Beatriz Joven-Ibáñez Speakers bureau: AbbVie, Celgene, Janssen, MSD, Novartis, Pfizer, Tatiana Korotaeva Speakers bureau: AbbVie, Amgen, Biocad, Janssen, Lilly, MSD, Novartis, Novartis-Sandoz, Pfizer, UCB, Consultant of: AbbVie, Amgen, Biocad, Janssen, Lilly, MSD, Novartis, Novartis-Sandoz, Pfizer, UCB, Grant/research support from: Pfizer, Wim Noel Employee of: Janssen, Petros Sfikakis Consultant of: AbbVie, Actelion, Boehringer Ingelheim, Enorasis, Farmaserv-Lilly, Genesis, Gilead, MSD, Novartis, Pfizer, UCB, Grant/research support from: AbbVie, Amgen, Boehringer Ingelheim, Faran, Janssen, Pfizer, Roche, Elke Theander Employee of: Janssen, Josef S. Smolen Speakers bureau: AbbVie, Amgen, AstraZeneca, Astro, Bristol-Myers Squibb, Celgene, Celltrion, Chugai, Gilead, ILTOO, Janssen, Lilly, MSD, Novartis-Sandoz, Pfizer, Roche, Samsung, Sanofi, UCB, Consultant of: AbbVie, AstraZeneca, Lilly, Novartis, Roche, Laure Gossec Consultant of: AbbVie, Amgen, Bristol-Myers Squibb, Biogen, Celgene, Galapagos, Gilead, Janssen, Lilly, Novartis, Pfizer, Samsung Bioepis, Sanofi-Aventis, UCB, Grant/research support from: Amgen, Galapagos, Janssen, Lilly, Pfizer, Sandoz, Sanofi

DOI: 10.1136/annrheumdis-2021-eular.1575

\section{OP0233 EFFICACY AND SAFETY OF UPADACITINIB IN PATIENTS WITH PSORIATIC ARTHRITIS AND AXIAL INVOLVEMENT}

A. Deodhar ${ }^{1}$, R. Ranza ${ }^{2}$, F. Ganz ${ }^{3}$, T. Gao ${ }^{4}$, J. Anderson ${ }^{4}$, A. Ostor ${ }^{5}$. ${ }^{1}$ Oregon Health \& Science University, Rheumatology, Portland, United States of America; ${ }^{2}$ Universidade Federal de Uberlândia, Rheumatology, Uberlândia, Brazil; ${ }^{3}$ AbbVie Inc., Rheumatology, Baar, Switzerland; ${ }^{4}$ AbbVie Inc., Rheumatology, North Chicago, United States of America; ${ }^{5}$ Cabrini Hospital, Monash University \& Emeritus Research, Melbourne, Australia

Background: Patients (pts) with psoriatic arthritis (PsA) and axial involvment exhibit greater disease activity and quality of life impairments compared with those without axial involvment

Objectives: To characterize PsA pts with and without axial involvement and compare efficacy of UPA vs placebo (PBO) in PsA pts with axial involvement.

Methods: In SELECT-PsA 1 (NCT03104400; N=1705, non-biologic DMARD IR) and SELECT-PsA 2 (NCT03104374; N=642, biologic DMARD IR), pts with active PsA ( $\geq 3$ swollen and $\geq 3$ tender joints), active or historical psoriasis, and on $\leq 2$ non-biologic DMARDs were randomized to once daily UPA $15 \mathrm{mg}$, UPA $30 \mathrm{mg}$, adalimumab $40 \mathrm{mg}$ every other week (SELECT-PsA 1 only), or PBO. Efficacy was assessed in pts with axial involvement (diagnosed by investigators based on totality of information) pooled from the 2 studies. Assessments included change from BL in BASDAI, BASDAI Q2 (neck/back/hip pain) and Q3 (joint swelling/pain), and the AS Disease Activity Score (ASDAS-CRP), and percentage with BASDAI 50 response, ASDAS inactive disease (ID), ASDAS low disease activity (LDA), ASDAS major improvement (MI), and ASDAS clinically important improvement (CII). Uveitis and inflammatory bowel disease (IBD) adverse events were reviewed. Data on 24-week PBO-controlled period are presented.

Results: Prevalence of axial involvment was $31.3 \%$ in SELECT-PsA 1 and $34.2 \%$ in SELECT-PsA 2 (Table). Treatment with UPA $15 \mathrm{mg}$ and $30 \mathrm{mg}$ resulted in significantly greater improvements from BL in the BASDAI, BASDAI Q2 (neck/back/ hip pain) and Q3 (joint swelling/pain) and ASDAS-CRP at weeks 12 and 24 vs PBO (Figure). Similarly, significantly higher percentages of pts on UPA $15 \mathrm{mg}$ and $30 \mathrm{mg}$ achieved BASDAI 50, ASDAS ID, LDA, MI, and Cll at weeks 12 and 24 vs PBO (Figure). One pt on UPA $30 \mathrm{mg}$ had incident uveitis, and no IBD was reported on UPA.

Conclusion: PsA pts with axial involvement had higher BL disease burden compared with those without axial involvement. UPA was efficacious in treating axial symptoms in pts with psoriatic spondylits.

REFERENCES:

[1] van der Heijde D, et al. Lancet. 2019;394(10214):2108-2117.

Acknowledgements: Abbvie funded the study. AbbVie participated in study design, research, analysis, data collection, interpretation of the data, reviewing, and approval. All authors had access to the relevant data and participated in the drafting, review, and approval of the abstract. No honoraria or payments were made for authorship. Medical writing support was provided by M Mehta, and J Matsuura of ICON plc (North Wales, PA) and was funded by AbbVie.
Table 1. Demographics and Baseline Characteristics

\begin{tabular}{|c|c|c|c|c|c|c|}
\hline \multirow[b]{2}{*}{$\begin{array}{l}\text { Parameter, } \\
\text { mean (SD) }\end{array}$} & \multicolumn{3}{|c|}{ SELECT-PsA 1} & \multicolumn{3}{|c|}{ SELECT-PsA 2} \\
\hline & $\begin{array}{c}\text { With } \\
\text { Psoriatic } \\
\text { Spondylitis } \\
(n=534)\end{array}$ & $\begin{array}{l}\text { Without } \\
\text { Psoriatic } \\
\text { Spondylitis } \\
(n=1170)\end{array}$ & $\begin{array}{c}P \\
\text { value }^{*}\end{array}$ & $\begin{array}{c}\text { With } \\
\text { Psoriatic } \\
\text { Spondylitis } \\
(n=219)\end{array}$ & $\begin{array}{l}\text { Without } \\
\text { Psoriatic } \\
\text { Spondylitis } \\
(n=421)\end{array}$ & $\begin{array}{c}P \\
\text { value }^{*}\end{array}$ \\
\hline BMI $\left(\mathrm{kg} / \mathrm{m}^{2}\right)$ & $29.9(6.5)$ & $30.5(6.9)$ & .0810 & $31.6(8.0)$ & $31.3(6.9)$ & .6226 \\
\hline TJC68 & $21.6(15.1)$ & $19.2(13.5)$ & .0022 & $27.5(18.0)$ & $23.3(16.2)$ & .0027 \\
\hline SJC66 & $11.7(9.4)$ & $11.0(7.9)$ & .1184 & $12.9(9.2)$ & $11.7(8.7)$ & .0804 \\
\hline $\begin{array}{l}\text { Physician's Global } \\
\text { Assessment } \\
\text { (NRS 0-10) }\end{array}$ & $6.7(1.6)$ & $6.5(1.7)$ & .0437 & $6.6(1.8)$ & $6.5(1.7)$ & .1897 \\
\hline HAQ-DI & $\begin{array}{c}1.2(0.6) \\
n=531\end{array}$ & $\begin{array}{l}1.1(0.6) \\
n=1164\end{array}$ & .0170 & $\begin{array}{c}1.2(0.6) \\
n=218\end{array}$ & $\begin{array}{c}1.2(0.7) \\
n=416\end{array}$ & .2049 \\
\hline $\begin{array}{l}\text { Presence of } \\
\text { dactylitis, } \\
\mathrm{n}(\%)^{\dagger}\end{array}$ & $188(35.2)$ & $328(28.0)$ & .0028 & $69(31.5)$ & $100(23.8)$ & .0348 \\
\hline $\begin{array}{l}\text { Presence of } \\
\text { enthesitis, } \\
\mathrm{n}(\%)^{\ddagger}\end{array}$ & $432(80.9)$ & $884(75.6)$ & .0147 & $189(86.3)$ & $337(80.0)$ & .0125 \\
\hline ASDAS-CRP & $\begin{array}{c}3.4(0.9) \\
n=530\end{array}$ & $\begin{array}{l}3.1(1.0) \\
n=1161\end{array}$ & $<.0001$ & $\begin{array}{c}3.3(1.0) \\
n=217\end{array}$ & $\begin{array}{c}3.2(1.1) \\
n=416\end{array}$ & .1032 \\
\hline BASDAI & $\begin{array}{c}5.8(2.0) \\
n=530\end{array}$ & $\begin{array}{c}5.3(2.2) \\
n=1161\end{array}$ & $<.0001$ & $\begin{array}{c}6.2(2.2) \\
n=217\end{array}$ & $\begin{array}{c}5.8(2.2) \\
n=416\end{array}$ & .0673 \\
\hline $\begin{array}{l}\text { Morning Stiffness } \\
\text { Duration (NRS } \\
0-10 ; \\
\text { BASDAI Q6) }\end{array}$ & $5.0(3.0)$ & $4.7(3.0)$ & .0368 & $5.6(3.2)$ & $5.1(3.0)$ & .0454 \\
\hline $\begin{array}{l}\text { Patient's } \\
\text { Assessment of } \\
\text { Inflammatory } \\
\text { Neck, Back, or Hip } \\
\text { Pain (NRS 0-10; } \\
\text { BASDAI Q2) }\end{array}$ & $\begin{array}{c}n=530 \\
5.8(2.7)\end{array}$ & $\begin{array}{c}n=1161 \\
4.6(3.2)\end{array}$ & $<.0001$ & $\begin{array}{c}n=217 \\
6.4(2.8)\end{array}$ & $\begin{array}{c}n=416 \\
5.4(3.1)\end{array}$ & .0001 \\
\hline & $\mathrm{n}=530$ & $\mathrm{n}=1161$ & & $\mathrm{n}=217$ & $\mathrm{n}=416$ & \\
\hline
\end{tabular}

${ }^{*}$ Calculated by t-test for continuous variables and chi-square test for categorical values. Bolded if $<0.05$. Defined as ${ }^{\dagger} \mathrm{LDI}>0$ and ${ }^{\ddagger}$ total enthesitis count $>0$

Figure. Integrated Analysis of Efficacy in PsA Patients With Axial Involvement

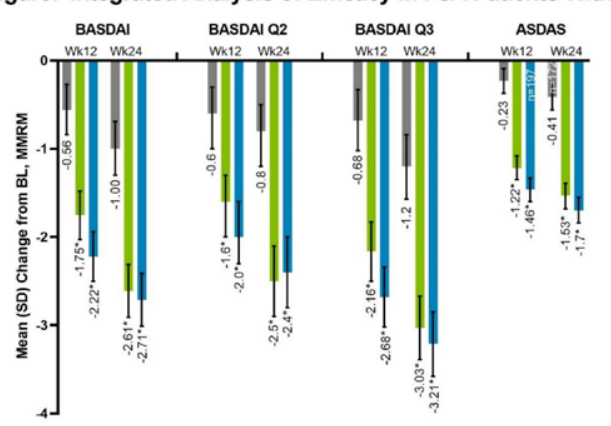

$=\begin{aligned} & \text { PBO } \\ & \text { (Wk 12/24: } n=186 / 174)\end{aligned} \underset{\text { UPA 15 mg QD }}{\text { (Wk 12/24: } n=203 / 186)}=\underset{\text { UPA } 30 \mathrm{mg} \text { QD }}{\text { (Wk 12/24: } n=198 / 181)}$
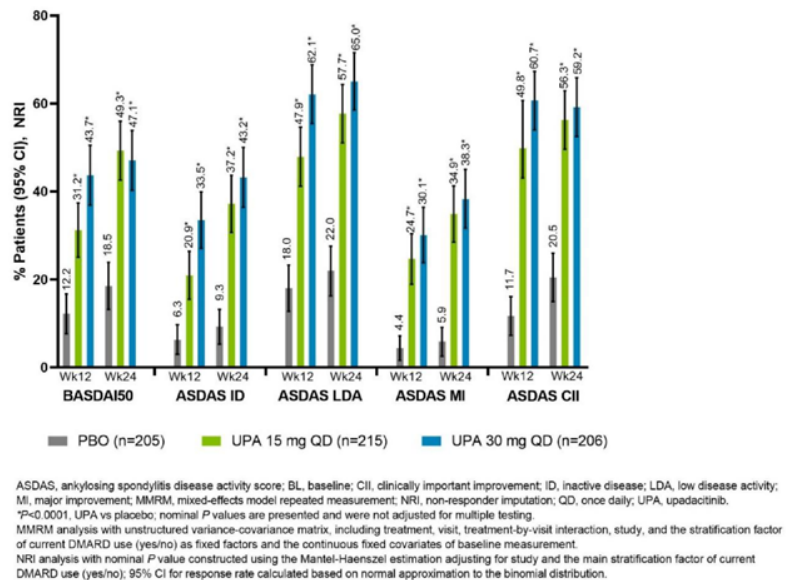
Disclosure of Interests: Atul Deodhar Speakers bureau: Novartis and Pfizer, Consultant of: Novartis, Pfizer, AbbVie, Eli Lilly, UCB Pharma, GlaxoSmithKline, Galapagos, Janssen, Boehringer Ingelheim and Celgene, Grant/research support from: Novartis, Pfizer, AbbVie, Eli Lilly, UCB Pharma, GlaxoSmithKline, R Ranza Speakers bureau: AbbVie, Janssen, Lilly, Novartis, and Pfizer, Consultant of: AbbVie, Janssen, Lilly, Novartis, and Pfizer, Grant/research support from: AbbVie, Janssen, Fabiana Ganz Shareholder of: AbbVie, Employee of: AbbVie, Tianming Gao Shareholder of: AbbVie, Employee of: AbbVie, Jaclyn Anderson Shareholder of: AbbVie, Employee of: AbbVie, Andrew Ostor Consultant of: AbbVie, BMS, Roche, Janssen, Lilly, Novartis, Pfizer, UCB, Gilead, and Paradigm DOI: 10.1136/annrheumdis-2021-eular.439

\section{Epidemiology and public health}

\section{OP0234 RISK OF ACUTE MYOCARDIAL INFARCTION AMONG NEW USERS OF CHONDROITIN SULPHATE: A NESTED CASE-CONTROL STUDY}

R. Mazzucchelli', S. Rodriguez-Martin ${ }^{2}$, A. García-Vadillo ${ }^{3}$,

M. Gil ${ }^{4}$, A. Rodríguez-Miguel ${ }^{2}$, D. Barreira-Hernández ${ }^{5}$, A. García-Lledó 6 , F. De Abajo ${ }^{7} .{ }^{1}$ Hospital Universitario Fundación Alcorcón, Rheumatologu Unit, Alcorcón, Spain; ${ }^{2}$ Hospital Universitario Príncipe de Asturias, Clinical Pharmacology Unit, Alcalá de Henares, Spain; ${ }^{3}$ Hospital de La Princesa, Rheumatologu Unit, Madrid, Spain; ${ }^{4}$ Spanish Agency of Medicines and Medical Devices, Division of Pharmacoepidemiology and Pharmacovigilance, Madrid, Spain; ${ }^{5}$ Hospital Universitario Príncipe de Asturias, Clinical Pharmacology Unit, Meco, Spain; ${ }^{6}$ Hospital Universitario Príncipe de Asturias, Department of Cardiology, Alcalá de Henares, Spain; ${ }^{7}$ Hospital Universitario Príncipe de Asturias, 2 Clinical Pharmacology Unit, Alcalá de Henares, Spain

Background: There is some evidence from epidemiological studies suggesting that CS and glucosamine could play a role in cardiovascular disease (CVD) prevention (1-4).Studies to date have included prevalent users, therefore a bias that overestimates protection cannot be excluded.

Objectives: To test the hypothesis that chondroitin sulphate (CS) or glucosamine reduce the risk of acute myocardial infarction (AMI).

Methods: Case-control study nested in a primary cohort composed of patients aged 40 to 99 years, with at least one year of follow-up in the BIFAP database during the 2002-2015 study period. From this cohort of patients, we identified incident cases of $\mathrm{AMI}$ and randomly selected five controls per case, matched by exact age, gender, and index date. Adjusted odds ratios (AOR) and their corresponding $95 \%$ confidence interval $(\mathrm{Cl})$ ) were calculated through a conditional logistic regression. Only new users of CS or glucosamine were considered.

Results: A total of 23,585 incident cases of AMI and 117,405 controls were included. The mean age was 67.0 (SD 13.4) years and $71.75 \%$ were male, in both groups. $558(2.37 \%)$ cases and 3,082 (2.62\%) controls used or had used $\mathrm{CS}$. The current use of CS was associated with a lower risk of AMI (AOR 0.57; 95\% Cl: 0.46-0.72) and disappeared after discontinuation (recent and past users). The reduced risk among current users was observed in both short-term (<365 days AOR $0.58 ; 95 \% \mathrm{Cl}: 0.45-0.75$ ) and long-term users ( $>364$ days AOR $0.56 ; 95 \% \mathrm{Cl} 0.36-0.87$ ), in both sexes (men, $\mathrm{AOR}=0.52$; $95 \% \mathrm{Cl}: 0.38-0.70$; women, $\mathrm{AOR}=0.65 ; 95 \% \mathrm{Cl}: 0.46-0.91$ ), in individuals over or under 70 years of age $(A O R=0.54 ; 95 \% \mathrm{Cl}: 0.38-0.77$, and $A O R=0.61 ; 95 \% \mathrm{Cl}: 0.45-0.82$, respectively) and in individuals at intermediate (AOR=0.65;95\%Cl:0.48-0.91) and high cardiovascular risk (AOR $=0.48 ; 95 \% \mathrm{Cl}: 0.27-0.83)$, but not in those at low risk (AOR=1.11;95\% Cl:0.48-2.56). In contrast, the current use of glucosamine was not associated with either increased or decreased risk of $\mathrm{AMI}(\mathrm{AOR}=0.86$; Cl95\% 0.66-1.08)

Conclusion: Our results support a cardioprotective effect of CS, while no effect was observed with glucosamine. The highest protection was found among subgroups at higher cardiovascular risk.

\section{REFERENCES:}

[1] Ma H, Li X, Sun D, Zhou T, Ley SH, Gustat J, et al. Association of habitual glucosamine use with risk of cardiovascular disease: prospective study in UK Biobank. BMJ. 2019;365(Journal Article):I1628.

[2] de Abajo FJ, Gil MJ, Garcia Poza P, Bryant V, Oliva B, Timoner J, et al. Risk of nonfatal acute myocardial infarction associated with non-steroidal antiinflammatory drugs, non-narcotic analgesics and other drugs used in osteoarthritis: a nested case-control study. PharmacoepidemiolDrug Saf. 2014;23(11):1128-38.

[3] Li Z-H, Gao X, Chung VC, Zhong W-F, Fu Q, Lv Y-B, et al. Associations of regular glucosamine use with all-cause and cause-specific mortality: a large prospective cohort study. Ann Rheum Dis. 2020 Apr 6;annrheumdis-2020-217176.

[4] King DE, Xiang J. Glucosamine/Chondroitin and Mortality in a US NHANES Cohort. J Am Board Fam Med. 2020 Dec;33(6):842-7.
Disclosure of Interests: Ramón Mazzucchelli Speakers bureau: UCB, Lilly, Grant/research support from: Pfizer, Roche, Amgen, Sara Rodriguez-Martin: None declared, Alberto García-Vadillo: None declared, Miguel Gil: None declared, Antonio Rodríguez-Miguel: None declared, Diana Barreira-Hernández: None declared, Alberto García-Lledó: None declared, Francisco de Abajo: None declared

DOI: 10.1136/annrheumdis-2021-eular.123

\section{OP0235 GOUT: 3 PROSPECTIVE COHORT STUDIES OF US MEN AND WOMEN}

N. McCormick ${ }^{1,2,3,4}$, C. Yokose ${ }^{1,2,3}$, L. Lu ${ }^{4}$, A. Joshi ${ }^{2,3,5}$, H. Choi ${ }^{1,2,3,4}$.

${ }^{1}$ Massachusetts General Hospital, Clinical Epidemiology Program, Division of Rheumatology, Allergy, and Immunology, Boston, United States of America; ${ }^{2}$ Harvard Medical School, Medicine, Boston, United States of America;

${ }^{3}$ Massachusetts General Hospital, The Mongan Institute, Boston, United States of America: ${ }^{4}$ Arthritis Research Canada, Richmond, Canada; ${ }^{5}$ Massachusetts General Hospital, Clinical \& Translational Epidemiology Unit, Boston, United States of America

Background: Emerging evidence suggests inflammation may drive progression from hyperuricemia to clinical gout, but the role of extrinsic, modifiable sources of chronic inflammation, such as diet, on gout risk is unknown. Notably, greater dietary inflammatory potential has been independently associated with increased risk of incident cardiovascular disease (CVD) ${ }^{1}$ and type 2 diabetes (T2D). ${ }^{2}$

Objectives: Prospectively examine the relation between dietary inflammatory potential and risk of gout in three large cohorts of US women and men over 30 years.

Methods: Ascertaining the ACR survey criteria for gout for several decades, ${ }^{3}$ we studied gout risk among 164,090 women from Nurses Health Study I (19862016) and II (1989-2017) and 40,598 men from Health Professionals Follow-up Study (1986-2016), free of gout at baseline. Dietary intake and covariates were assessed by validated questionnaires every 4 years. Inflammatory potential of diet was evaluated using a food-based empirical dietary index of inflammatory potential score (EDIP) pre-defined based on circulating levels of IL-6, C-reactive protein, adiponectin, and TNF $\alpha$ R2. ${ }^{4}$

We assigned an EDIP score for each participant, adjusted for total energy take, and prospectively examined the association between quintiles of EDIP score and incident gout, adjusting for potential confounders. We also stratified by alcohol intake, as alcohol has anti-inflammatory properties, ${ }^{4}$ but is associated with a higher gout risk, particularly beer. ${ }^{5}$

Results: We documented 2,874 incident gout cases over 5,124,940 person-years of follow-up. In pooled multivariable-adjusted analyses, those in the highest EDIP quintile had 59\% higher gout risk (multivariable RR 1.59; $95 \% \mathrm{C}$ 1.41-1.79), compared with the lowest (Table 1). This remained positive with further adjustment for BMI, a likely causal intermediate (RR 1.27, 1.12 to 1.42), and was stronger among non-drinkers (RR 2.37, 1.58 to 2.56 ) than drinkers (RR 1.57, 1.38 to 1.78 ) (Table 1 ).

Table 1. Risk Ratio (95\% Cl) of Gout According to Quintiles of Inflammatory Diet Score, Overall and by Alcohol Use

\begin{tabular}{|c|c|c|c|c|c|c|}
\hline & $\begin{array}{l}\text { Q1: } \\
\text { lowest }\end{array}$ & Q2 & Q3 & Q4 & $\begin{array}{c}\text { Q5: } \\
\text { highest }\end{array}$ & $\begin{array}{l}P \text { for } \\
\text { trend }\end{array}$ \\
\hline \multicolumn{7}{|l|}{ Overall } \\
\hline $\mathrm{N}$ cases & 473 & 493 & 530 & 623 & 755 & \\
\hline Person-years & $1,024,571$ & $1,025,618$ & $1,025,284$ & $1,024,779$ & $1,024,688$ & \\
\hline Age-adjusted RR & 1.00 (Ref) & $\begin{array}{c}1.05(0.92 \\
1.19)\end{array}$ & $\begin{array}{c}1.13(0.99 \\
1.27)\end{array}$ & $\begin{array}{c}1.33(1.18 \\
1.50)\end{array}$ & $\begin{array}{c}1.64(1.46 \\
1.84)\end{array}$ & $<0.001$ \\
\hline MV-Adjusted RR & 1.00 (Ref) & $\begin{array}{c}1.04(0.92 \\
1.18)\end{array}$ & $\begin{array}{c}1.12(0.98 \\
1.26)\end{array}$ & $\begin{array}{c}1.31(1.16 \\
1.48)\end{array}$ & $\begin{array}{c}1.59(1.41 \\
1.79)\end{array}$ & $<0.001$ \\
\hline $\begin{array}{l}\text { MV-Adjusted }{ }^{\star \star} \text { RR } \\
\text { (+ BMI) } \\
\text { No Alcohol Use }\end{array}$ & 1.00 (Ref) & $\begin{array}{c}1.00(0.88 \\
1.13)\end{array}$ & $\begin{array}{c}1.03(0.91 \\
1.17)\end{array}$ & $\begin{array}{c}1.16(1.02 \\
1.31)\end{array}$ & $\begin{array}{l}1.27(1.12 \\
1.42)\end{array}$ & $<0.001$ \\
\hline $\mathrm{N}$ cases & 26 & 58 & 84 & 143 & 251 & \\
\hline Person-years & 118,301 & 189,938 & 249,389 & 313,511 & 396,080 & \\
\hline MV-Adjusted RR & 1.00 (Ref) & $\begin{array}{c}1.31(0.82 \\
2.08)\end{array}$ & $\begin{array}{c}1.37(0.88 \\
2.13)\end{array}$ & $\begin{array}{c}1.80(1.18 \\
2.74)\end{array}$ & $\begin{array}{c}2.37(1.58, \\
2.56)\end{array}$ & $<0.001$ \\
\hline $\begin{array}{l}\text { MV-Adjusted } \\
\text { RR (+ BMI) } \\
\text { Alcohol Use }\end{array}$ & 1.00 (Ref) & $\begin{array}{l}1.28(0.80 \\
2.03)\end{array}$ & $\begin{array}{c}1.32(0.85 \\
2.05)\end{array}$ & $\begin{array}{c}1.61(1.06 \\
2.45)\end{array}$ & $\begin{array}{l}1.85(1.23 \\
2.79)\end{array}$ & $<0.001$ \\
\hline $\mathrm{N}$ cases & 447 & 435 & 446 & 480 & 504 & \\
\hline Person-years & 906,271 & 835,680 & 775,895 & 711,267 & 628,609 & \\
\hline MV-Adjusted RR & 1.00 (Ref) & $\begin{array}{c}1.04(0.91 \\
1.19)\end{array}$ & $\begin{array}{c}1.13(0.99, \\
1.29)\end{array}$ & $\begin{array}{c}1.31(1.15 \\
1.50)\end{array}$ & $\begin{array}{c}1.57(1.38 \\
1.78)\end{array}$ & $<0.001$ \\
\hline $\begin{array}{l}\text { MV-Adjusted }{ }^{\star \star} \text { RR } \\
\quad \text { (+ BMI) }\end{array}$ & 1.00 (Ref) & $\begin{array}{c}1.00(0.88 \\
1.14)\end{array}$ & $\begin{array}{c}1.05(0.92 \\
1.20)\end{array}$ & $\begin{array}{c}1.17(1.03, \\
1.33)\end{array}$ & $\begin{array}{c}1.28(1.12 \\
1.46)\end{array}$ & $<0.001$ \\
\hline
\end{tabular}

*Multivariable (MV) models adjusted for age (month), White race, smoking, menopause (women only), hormone use (women only), physical activity, history of hypertension, and diuretic use. ${ }^{\star \star} \mathrm{MV}+\mathrm{BMI}$ models additionally adjusted for BMI (a likely causal intermediate) 